Article

\title{
Structural Tunable Plasma Photonic Crystals in Dielectric Barrier Discharge
}

\author{
Kuangya Gao $₫$, Yueqiang Liang, Chengyu Liu, Yafeng He, Weili Fan * and Fucheng Liu * \\ College of Physics Science and Technology, Hebei University, Baoding 071002, China; \\ gkyplasma@163.com (K.G.); liangyueqiangband@163.com (Y.L.); lcyplasma1056@163.com (C.L.); \\ heyf@hbu.edu.cn (Y.H.) \\ * Correspondence: fanweili@hbu.edu.cn (W.F.); hdlfc@hbu.edu.cn (F.L.); Tel.: +86-312-5079354 (W.F.)
}

Received: 9 January 2020; Accepted: 10 August 2020; Published: 12 August 2020

\begin{abstract}
We demonstrate a kind of structural tunable plasma photonic crystal in a dielectric barrier discharge by self-organization of the plasma filaments. The symmetry, the lattice constant and the orientations of different plasma photonic crystals can be deliberately controlled by changing the applied voltage. The plasma structures can be tuned from a square lattice to a triangular lattice, the lattice constant is reduced and the crystal orientation varies $\frac{\pi}{6}$ when the applied voltage is increased. The band diagrams of the plasma photonic crystals under a transverse-magnetic wave have been studied, which shows that the positions and sizes of the band gaps change significantly for different plasma structures. We suggest a flexible way for the fabrication of tunable plasma photonic crystals, which may find wide application in the manipulation of microwaves or terahertz waves.
\end{abstract}

Keywords: dielectric barrier discharge; plasma photonic crystals; band diagrams

\section{Introduction}

Photonic crystals can be used to selectively reflect specific frequencies of electromagnetic radiation. It is a significant tool to control the propagation of electromagnetic (EM) waves, which has been widely used in Bragg mirrors, optical waveguide, optical switch, solar cells, sensors and light-emitting diodes (LEDs) [1-10]. In recent years, there appears to have been great interest in fabricating tunable photonic crystals for real-time and on-demand control of the photonic band structures [11,12]. One of the most interesting applications of tunable photonic crystals is in designing novel sensing devices, such as highly sensitive temperature and refractive index sensors [9,10]. So far, different methods of producing reconfiguring photonic crystals have been demonstrated, including mechanical, thermal, biological, and opto-fluidic methods [13-16]. As a new kind of tunable photonic crystal, the plasma photonic crystal (PPC) has attracted considerable attention recently. It is a periodic structure with the plasma and dielectric material or the spatially periodic arrangement of the plasma density $[17,18]$. The PPCs not only have characteristic band gaps as in the conventional photonic crystals, but also possess a number of unique features such as the strong dispersion around the electron plasma frequency and the dynamic time-varying controllability $[19,20]$. Depending on the incident EM wave frequency and electron density, the relative permittivity of the plasmas can be tuned from strongly negative to positive values of less than unity (passing through zero) [21,22]. Moreover, it affords the possibility of rapidly controlling the band gap at rates determined only by the time to form or recombine the plasma [22]. These superior properties lead to development of dynamic and functional devices for EM waves ranging from microwaves to terahertz $(\mathrm{THz})$ waves, which can find wide applications in microwave filter, optical switch, wireless communications, millimeter-wave spectroscopy, plasmas stealth aircraft and so on [23-26]. 
To date, great efforts have been made in fabricating different types of PPCs [22,27-34]. The first type is a periodic array of plasma rods that are immersed in a gas or a solid background. Sakai et al., used a square array of metal lattice electrodes to generate periodic plasmas columns [27]. Ouyang et al., and Wang et al., used an array of gas discharge tubes to obtain one-dimensional and two-dimensional plasma photonic crystals, respectively [22,28]. Khalkhaliet et al., demonstrated a dielectric-plasma photonic crystal which is composed of plasma rods in the anisotropic tellurium background [29]. The second type of the PPCs consists of solid rods (dielectric or metal) forming a lattice which is immersed in a plasma background. Chaudhari et al., suggested a form of plasma metallic photonic crystals (PMPC) by inserting a periodic arrangement of metal rods into the plasma background [30]. Chern et al., studied one- and two-dimensional PMPCs with different geometries of the metal rods [31]. The third type of the PPC is realized by incorporating a functionalized plasma element into the photonic crystal. Wang et al., integrated the gaseous plasma elements into a microwave photonic crystal cavity structure to control the transmission properties [32]. All of these works contribute significantly to a rich conceptual framework of PPCs. In these studies, the tunable responses of PPCs are generally achieved by changing the plasma density or lattice constant of the plasma structures. However, it is still a challenge to fabricate more flexible plasma photonic crystals, whose geometric configuration including the symmetry, the lattice constant and the crystal orientation can all be conveniently modulated. This will provide additional tunability and find more practical applications for PPCs.

In this paper, we provide an efficient method to realize a rich variety of reconfigurable plasma photonic crystals in dielectric barrier discharge (DBD) by self-organization of the plasma filaments. The symmetry, the lattice constant and the orientations of different plasma photonic crystals can be deliberately changed by simply varying the applied voltage. In particular, these plasma structures can be rapidly reconstructed, which allow for active control within seconds or minutes. Based on the experimental results, the band diagrams of different plasma photonic crystals under a transverse-magnetic (TM) wave have been studied and characterized. The results would offer inspiration for engineering novel tunable optical materials with exploitable properties and find broad applications in the manipulation of microwaves or terahertz waves.

\section{Experimental Setup}

The schematic diagram of the experimental setup is illustrated in Figure 1. Two cylindrical containers with diameter of $7.5 \mathrm{~cm}$ filled with tap water were used as the electrodes. The water also served as a coolant and transparent media for observation and measurement of discharge filaments. The water electrodes were sealed with the glass plates with a thickness of $1.5 \mathrm{~mm}$. A metallic ring was immersed in each container and connected to a power supply. A sinusoidal alternating current (AC) voltage at the frequency $10-100 \mathrm{kHz}$ was applied to the left electrode and the right electrode was grounded. A square glass spacing was clamped between two parallel electrodes, serving as a lateral boundary, which determines the distance of the gas gap. The whole apparatus was enclosed in a big container filled with the static gas of different components and different pressures. The voltage waveform and current waveform were detected using a high-voltage probe (Tektronix P6015A 1000x) and a current probe (Tektronix TCP0030A), respectively, which were recorded using a digital phosphor oscilloscope (Tektronix TDS3054B). A camera (EOS 6D) was placed at one end of the electrode to record the images of PPCs. 


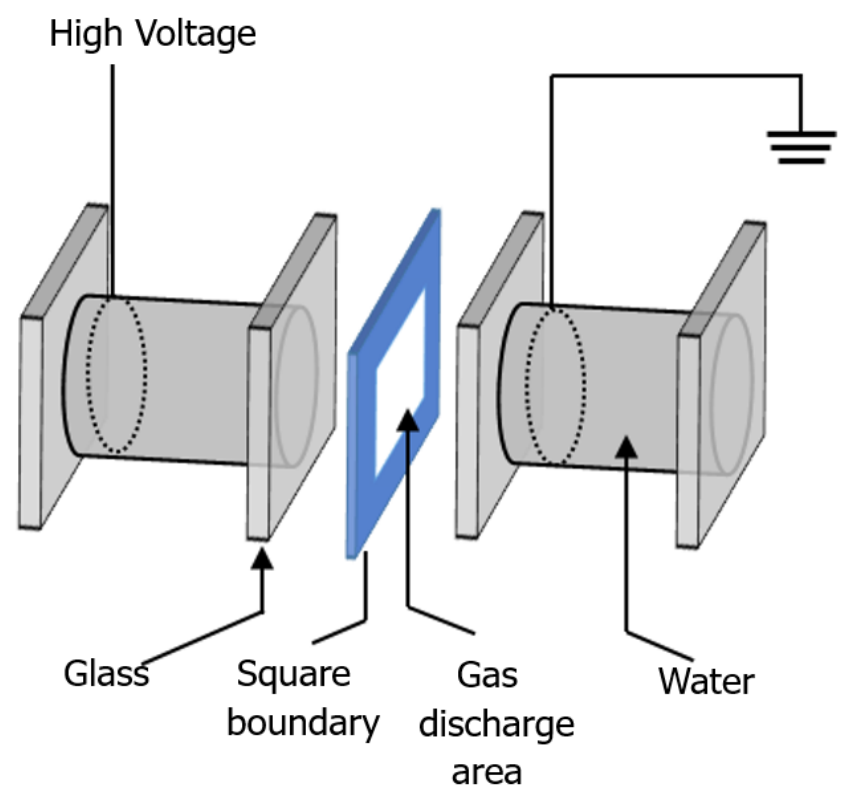

Figure 1. Schematic diagram of the experimental setup.

\section{Results and Discussion}

We started with the symmetry modulation of the plasma photonic crystals from a triangular lattice to a square lattice when increasing the applied voltage, as shown in Figure 2. These plasma structures are produced in pure air at atmospheric pressure, which are cost-effective and provide stirring potential in real applications. As displayed in Figure 2a, when the voltage is low at $U=5.3 \mathrm{kV}$, some bright filaments are ignited and they self-organize into a triangular lattice with the lattice constant $a=1.5 \mathrm{~mm}$. With an increase of the applied voltage, the triangular structure becomes unstable and it will transit to a well-defined square lattice with the lattice constant $a=1.2 \mathrm{~mm}$, as shown in Figure $2 \mathrm{~d}$. The symmetrical deformation can also be clearly identified in the spatial Fourier spectra as shown in Figure $2 \mathrm{~b}$,e. The voltage-current waveforms associated with different plasma structures are demonstrated in Figure 2c-f. One can see that a group of current spikes are produced with the time interval about several microseconds in each half cycle of the applied voltage. Moreover, the discharge density and discharge interval are increased by increasing the applied voltage. It is worth pointing out here that the plasma lattices as shown in Figure $2 \mathrm{a}, \mathrm{d}$ are complete and stable, which are actually the time-averaged structures over thousands of AC cycles. The time-resolved measurements of these plasma structures with sub-microsecond scale have been performed, which are not presented here. The results indicate instantly that the crystal lattice is not so perfect and some vacancies may occur, which is caused by the temporal shifts of the micro-discharges living several tens of nanoseconds while forming a lattice within the discharge interval about several microseconds long. The dynamical plasma structures composed of different spatiotemporal sublattices that are ignited at different time instants were also observed in our previous studies [35-37]. In the preliminary work presented here, we did not take account of these complicated spatio-temporal behaviors, and the changes of the band diagrams are examined when the time-averaged structures of the plasma photonic crystals have been changed. Similar plasma lattices were observed by Matlis et al., with similar discharge conditions to our experiments [38]. Band gaps between 17.5-20.0 GHz were detected and the anisotropic behavior of the plasma lattices was observed. It is proved that such plasma lattices could be used as a promising material to manipulate the propagation of microwaves. 

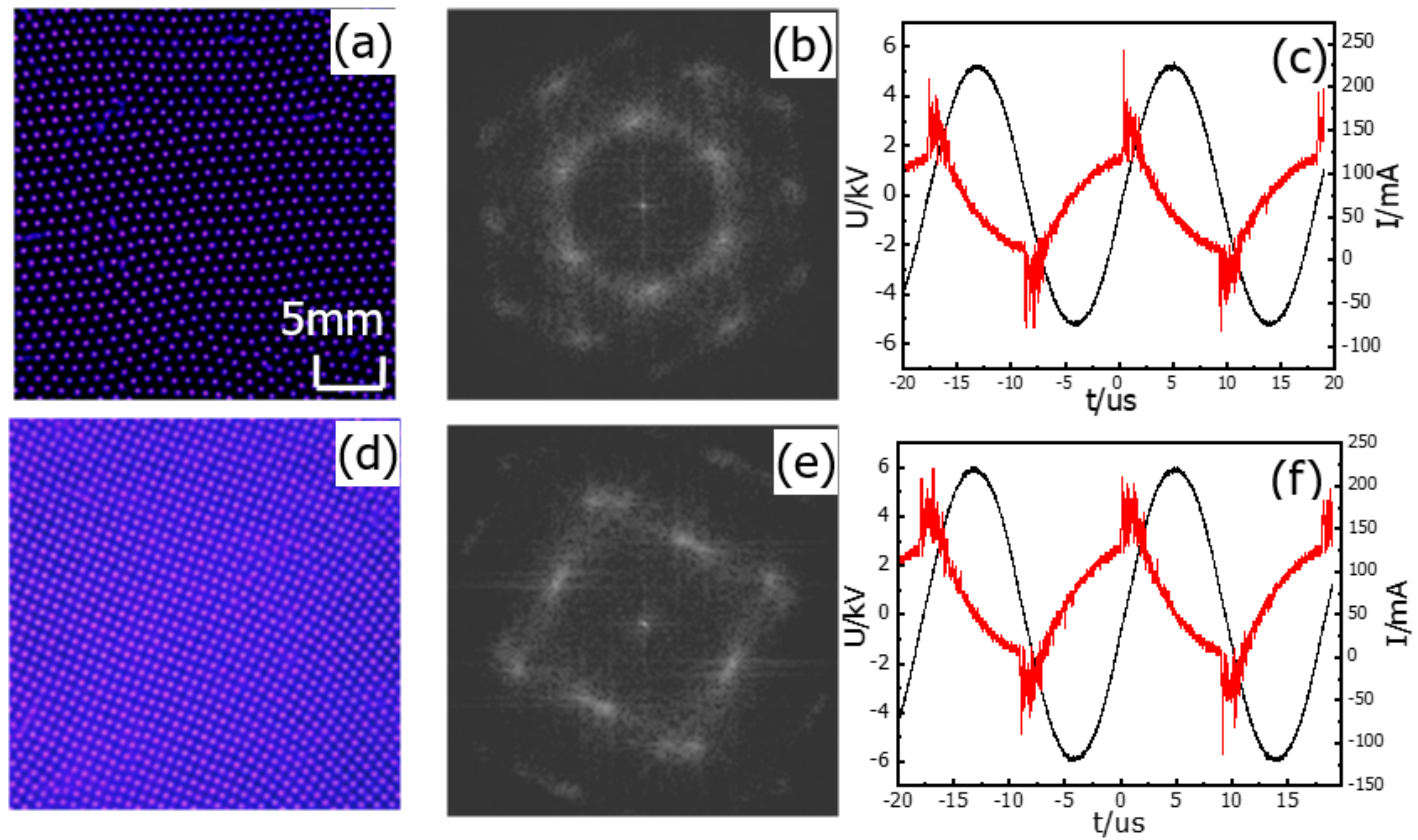

Figure 2. Plasma photonic crystals with different symmetries when increasing of the applied voltage. The left column: snapshots of the triangular plasma photonic crystal (a) and square plasma photonic crystal (d). The middle column: the corresponding Fourier spectra of triangular lattice (b) and square lattice (e). Right column: the voltage-current waveforms associated with the triangular lattice (c) and square lattice (f). The discharge parameters are: (a) the applied voltage $U=5.3 \mathrm{kV}$, discharge power $p=32 \mathrm{~W}(\mathrm{~d}) U=6.0 \mathrm{kV}, p=46 \mathrm{~W}$. The frequency of the applied voltage $f=55 \mathrm{kHz}$. The working gas is $100 \%$ air. The distance of the gas gap $d=1.1 \mathrm{~mm}$. Gas pressure $p=300$ torr and the exposure time $t=40 \mathrm{~ms}$.

Figure 3 presents the schematic views of the triangular lattice and the square lattice as well as their corresponding irreducible Brillouin zones. The plasma photonic crystal consists of circular plasma columns immersed in air with the dielectric constant $\varepsilon_{\mathrm{a}}=1$. For the plasmas, its dielectric constant is frequency-dependent, which can be written as:

$$
\varepsilon_{p}(\omega)=1-\frac{\omega_{\text {pe }}^{2}}{\omega\left(\omega-j v_{m}\right)}
$$

where $\omega_{\mathrm{pe}}, v_{\mathrm{m}}$, and $\omega$ are the plasma frequency, the electron collision frequency, and the frequency of the incident EM waves, respectively. $\omega_{p e}=\left(e^{2} n_{e} / \varepsilon_{0} m\right)^{1 / 2}$ where $n_{e}$ is the electron density, which can be measured by the spectrum method as discussed in our previous papers [39,40]. In general, the electron density $\mathrm{n}_{\mathrm{e}}$ is increased by increasing of the applied voltage and the gas pressure. For the discharges operated in a mixture of Ar/air, $\mathrm{n}_{\mathrm{e}}$ increases with the content of air. The electron collision frequency $v_{\mathrm{m}}=\mathrm{N} \delta_{\mathrm{C}}(v) v$, where $v$ is the speed of the electrons, $\mathrm{N}$ is the density of neutral atoms per unit volume, and $\sigma_{C}(v)$ is the collision cross section between the electrons and neutrals. The real part of the relative dielectric constant $\varepsilon_{\mathrm{p}}$ given in Equation (2) is used to calculate the dispersion relations of the plasma photonic crystals, while the imaginary part is responsible for dissipation and absorption of the EM waves, which is not taken into account here.

$$
\operatorname{Re}\left(\varepsilon_{\mathrm{p}}\right)=1-\frac{\omega_{\mathrm{pe}}^{2}}{\omega^{2}+v_{\mathrm{m}}^{2}}
$$




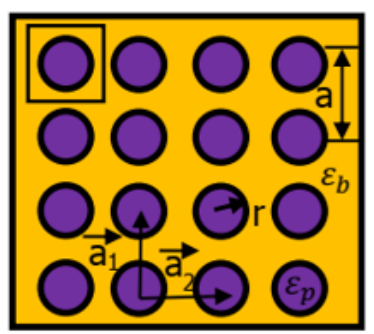

(a)

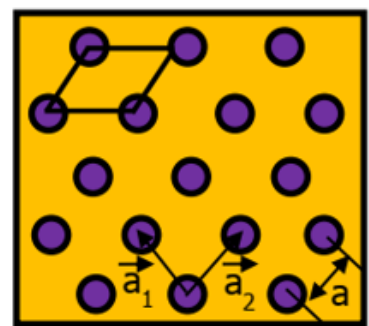

(c)

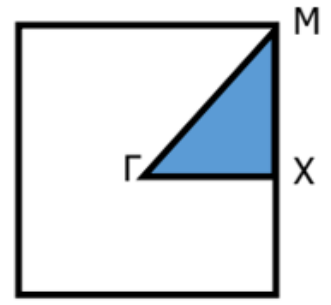

(b)

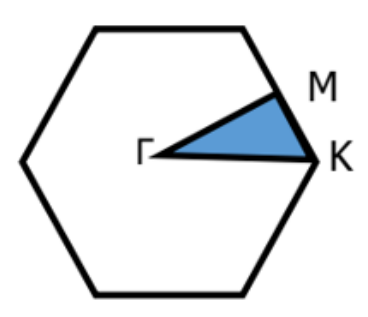

(d)

Figure 3. Schematics of a square lattice (a) and a triangular lattice (c) and the corresponding reduced Brillouin zone boundaries (b-d). Here a is the lattice constant; $r$ is the radius of the plasma column; $\mathrm{a}_{1}, \mathrm{a}_{2}$ denote the base vectors of the lattices; $\varepsilon_{\mathrm{a}}$ and $\varepsilon_{\mathrm{p}}$ indicate the dielectric constants of the gas and plasmas, respectively. The areas enclosed by the black and rhombus at the top left of $(\mathbf{a}, \mathbf{c})$ indicate the basic unit of the plasma photonic crystals. In figures $(\mathbf{b}, \mathbf{d})$, the triangular regions surrounded by $\mathrm{M}-\Gamma-\mathrm{X}-\mathrm{M}$ and $\mathrm{M}-\Gamma-\mathrm{K}-\mathrm{M}$ are the irreducible Brillouin regions which are utilized for calculating of the dispersion relations.

Simulations of the plasma photonic crystals with different configurations were completed by use of COMSOL Multiphysics software based on the finite element method. The Lorentz-Drude model was employed. The mesh division is selected as a free triangle mesh, and the largest mesh element is set as $8.33 \times 10^{-5} \mathrm{~m}$, the curvature factor is 0.25 . The areas enclosed by the black and rhombus in Figure $3 \mathrm{a}, \mathrm{c}$ indicate the basic units of different plasma photonic crystals. Floquet periodic boundary conditions are applied at two sets of opposite edges of the square and rhombus. According to Bloch's theorem, the eigenvalue frequencies corresponding to different wave vectors $\mathrm{k}$ along the irreducible Brillouin zone boundaries $\mathrm{M}-\Gamma-\mathrm{X}-\mathrm{M}$ and $\mathrm{M}-\Gamma-\mathrm{K}-\mathrm{M}$ are calculated, respectively. By scanning the coefficient $\mathrm{k}$ through the parameterized sweep built in COMSOL, the dispersion relations of EM waves for different plasma structures can be obtained.

Figure 4 illustrates the band diagrams of the triangular and the square plasma photonic crystals corresponding to the plasma structures shown in Figure 2. Due to the facts that there may have fluctuations of electron densities in the ensemble of filaments forming the crystal lattice, the band diagrams under different electron densities are studied. For similar conditions to the present experiments, $10^{13} \leq \mathrm{n}_{\mathrm{e}} \leq 10^{16} \mathrm{~cm}^{-3}$ [36,38-40]. Obviously, one can see that an increase of the electron density leads to formation of more band gaps for both of the square and triangular plasma photonic crystals. Moreover, the positions of the band gaps move to the higher frequency and the sizes of the band gaps are increased remarkably. Here we take the band diagram at the electron density $\mathrm{n}_{\mathrm{e}}=10^{15} \mathrm{~cm}^{-3}$ for example, which order of magnitude most commonly occurs in the discharges $[39,40]$. We can see that three unidirectional band gaps form for the triangular lattice as shown in Figure 4c. They locate at the position of $0.58-0.76 \mathrm{\omega a} / 2 \pi \mathrm{c}$ in $\mathrm{M}-\Gamma$ direction, and at $0.74-0.99 \omega \mathrm{wa} / 2 \pi \mathrm{c}$ and $1.08-1.26 \mathrm{\omega a} / 2 \pi \mathrm{c}$ in K-M direction, respectively. When the plasma photonic crystal is tuned to the square lattice by increasing the applied voltage as shown in Figure 4g, a remarkably different band structure forms. An absolute band gap is produced at the position of $0.72-0.77 \omega \mathrm{a} / 2 \pi \mathrm{c}$, and the EM waves within this frequency range cannot pass through the plasma crystals. Consequently, with the symmetrical 
deformation from the triangular lattice to square lattice, the band structures change significantly, which can be utilized to modulate the propagation of EM waves of different frequencies.
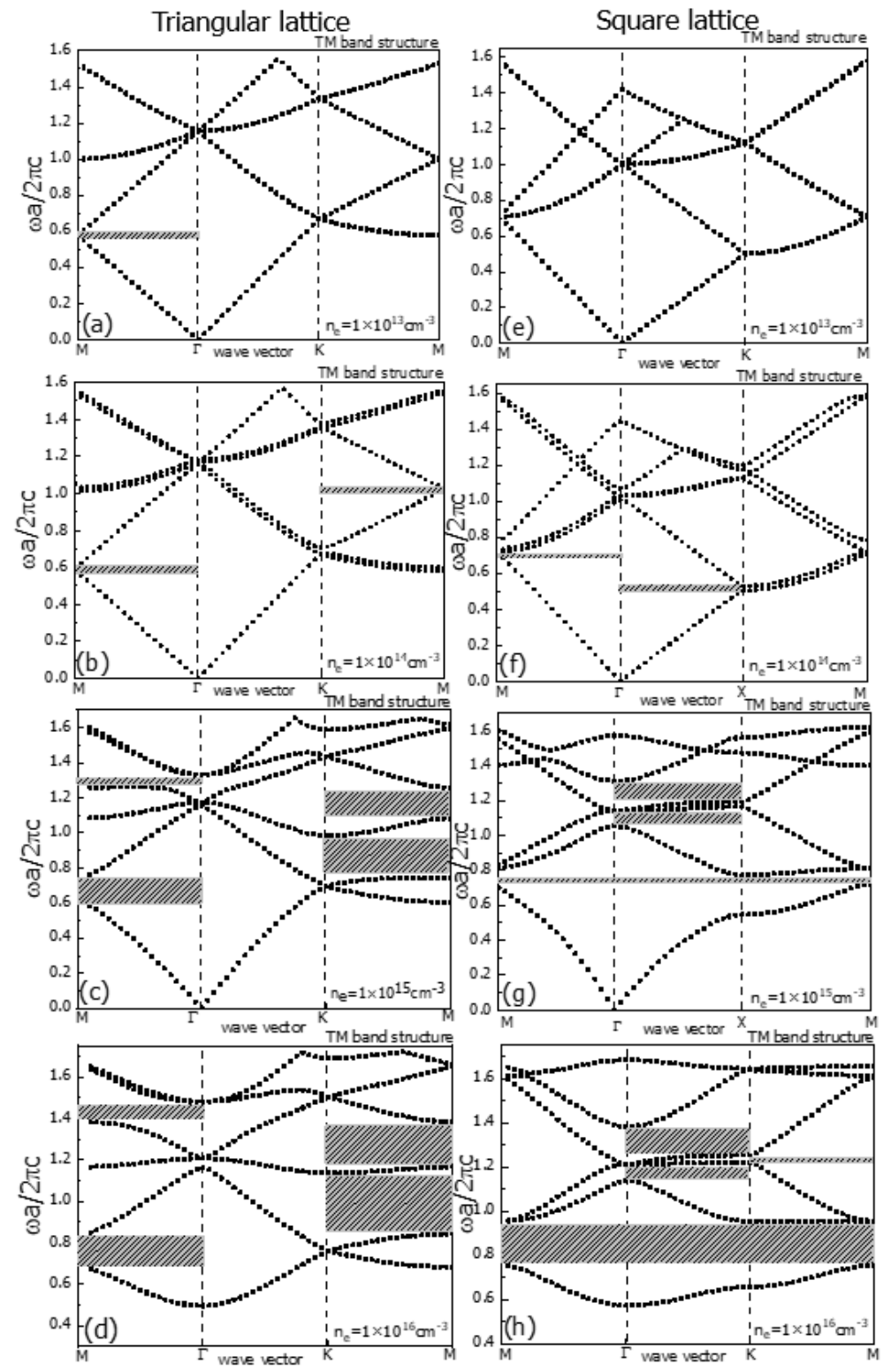

Figure 4. The dispersion relations under different electron densities for the triangular lattice (a-d) and the square lattice (e-h) shown in Figure $2 \mathrm{a}, \mathrm{b}$. The shadow strips indicate the positions of the band gaps. The plasma collision frequency $v_{\mathrm{m}}=160 \mathrm{GHz}$.

By changing the applied voltage, the lattice constant of the plasma structures can be dynamically controlled. As shown in Figure 5, the triangular lattices with different lattice constants are obtained in our experiment. More filaments are ignited and the lattice constants decrease by increasing the applied voltage. All these plasma structures are very stable, which can last for tens of minutes unless the discharge parameters are changed. To quantitatively characterize the spatial order of the plasma structures, the pair correlation functions (PCF) are calculated from the discharge density distributions. The PCFs have been used to characterize complex plasma crystals to show the probability density $\left(\rho_{\mathrm{r}}\right)$ to find a filament at distance $r$ from a given filament [41]. One can clearly see that the pair correlation function displays a series of isolated peaks, in which the first peak is the strongest, giving the value of the lattice constant. The lattice constant $a=2.68 \mathrm{~mm}, 2.59 \mathrm{~mm}, 2.48 \mathrm{~mm}$, respectively, which decreases 
with an increase of the applied voltage. The positions of $\rho_{\mathrm{r}}$ peaks for the ideal triangular lattices are given by the red short lines at the bottom of Figure $5 \mathrm{~d}-\mathrm{f}$. Obviously, they matched well with the $\rho_{\mathrm{r}}$ peaks of the plasma structures obtained in our experiment, indicating that these plasma lattices are very regular and close to the ideal triangular lattices. The voltage-current waveforms for different plasma photonic crystals are illustrated in Figure $5 \mathrm{~g}-\mathrm{i}$. One can see that more current spikes appear with an increase of the applied voltage, owing to the formation of more discharge filaments. As expected, the band diagrams change correspondingly when the lattice constant is reduced with increasing of the applied voltage. One can find in Figure $5 j$ that a complete band gap is produced when $a=2.68 \mathrm{~mm}$, which locates at the position of 1.18-1.22 wa/2 $2 \pi \mathrm{c}$. Besides, two unidirectional band gaps form in the $\mathrm{M}-\Gamma$ direction at the positions of $0.74-0.90 \omega \mathrm{a} / 2 \pi \mathrm{c}$ and $1.47-1.63 \omega \mathrm{a} / 2 \pi \mathrm{c}$, respectively, and two unidirectional band gaps are produced in the K-M direction at the positions of $1.23-1.47 \mathrm{wa} / 2 \pi \mathrm{c}$ and $1.68-1.76 \mathrm{wa} / 2 \pi \mathrm{c}$. When the lattice constant is reduced from $2.68 \mathrm{~mm}$ to $2.48 \mathrm{~mm}$, as shown in Figure $5 \mathrm{l}$, the width of the complete band gap becomes larger and its position is changed to $1.19-1.27 \mathrm{\omega a} / 2 \pi \mathrm{c}$. In addition, the positions of the other four unidirectional band gaps are also changed, which locate at $0.78-0.94 \omega \mathrm{a} / 2 \pi \mathrm{c}$ and $1.51-1.71 \omega \mathrm{a} / 2 \pi \mathrm{c}$ in the $\mathrm{M}-\Gamma$ direction and at $1.28-1.52 \omega \mathrm{a} / 2 \pi \mathrm{c}$ and $1.71-1.82 \omega \mathrm{a} / 2 \pi \mathrm{c}$, in the K-M direction, respectively. Obviously, the photonic bands move towards higher frequencies with a decrease of the lattice constant and the size of each band gap is increased. Thus, by changing the lattice constant, the plasma photonic crystals can be used to selectively control the propagation of EM waves of different frequencies.
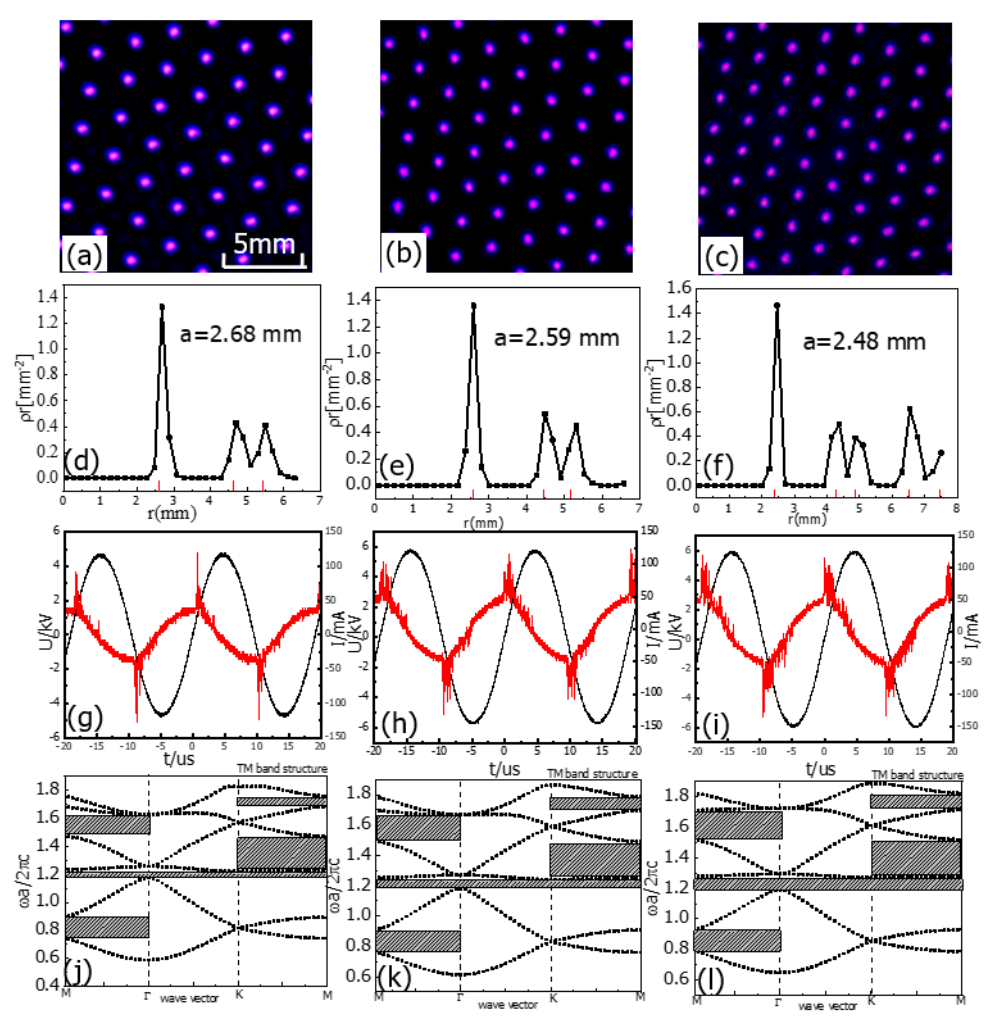

Figure 5. Plasma photonic crystals with reduced lattice constant when increasing of the applied voltage $(\mathbf{a}-\mathbf{c})$, the corresponding pair correlation functions $(\mathbf{d}-\mathbf{f})$, the voltage-current waveforms $(\mathbf{g}-\mathbf{i})$, and the corresponding band diagrams ( $\mathbf{j}-\mathbf{1})$. The discharge parameters are: (a) $U=4.8 \mathrm{kV}, p=14 \mathrm{~W}$ (b) $U=5.8 \mathrm{kV}, p=30 \mathrm{~W}$ (c) $U=6.0 \mathrm{kV}, p=34 \mathrm{~W} . f=53 \mathrm{kHz}, p=230$ torr, $d=2.0 \mathrm{~mm}, t=40 \mathrm{~ms}$. The working gas is $40 \% \mathrm{Ar}$ and $60 \%$ air. The red short lines at the bottom of $(\mathbf{d}-\mathbf{f})$ indicate the positions of $\rho_{\mathrm{r}}$ peaks for ideal triangular lattices. The electron density is set as $10^{15} \mathrm{~cm}^{-3}[36,38-40], v_{\mathrm{m}}=60 \mathrm{GHz}$.

Next, we examined the angular reorientation of the plasma photonic crystals. As shown in Figure $6 a, b$, the plasma crystals rotate $\frac{\pi}{6}$ when slightly increasing the applied voltage, while the 
other structural parameters are nearly invariant. Figure $6 c, d$ present the voltage-current waveforms corresponding to different plasma structures. One can see that a group of discharge spikes are produced, which occur at both of the falling and rising edge of the applied voltage (passing through the zero-crossing point of the ac voltage waveform). The time intervals of the discharges are about 6.0 us. The discharges occurring at the falling edge of the applied voltage are mainly dominated by the fields of surface charges, while the discharges occurring at the rising edge are initiated by the applied voltage [37]. The voltage-current waveforms change little when the orientation of the plasma has been changed. To some extent, the changes of the crystal orientation are equivalent to varying the incident angle of the EM waves, which may have significant influence on the photonic band diagrams. It was proven in previous studies that a blue shift of the photonic bands as well as a narrowed band gap is achieved when increasing the incident angle of the EM waves [42]. Thus, through modulating the orientation of the plasma structures, we suggest an alternative way to control the reflection and transmission behavior of the EM waves.
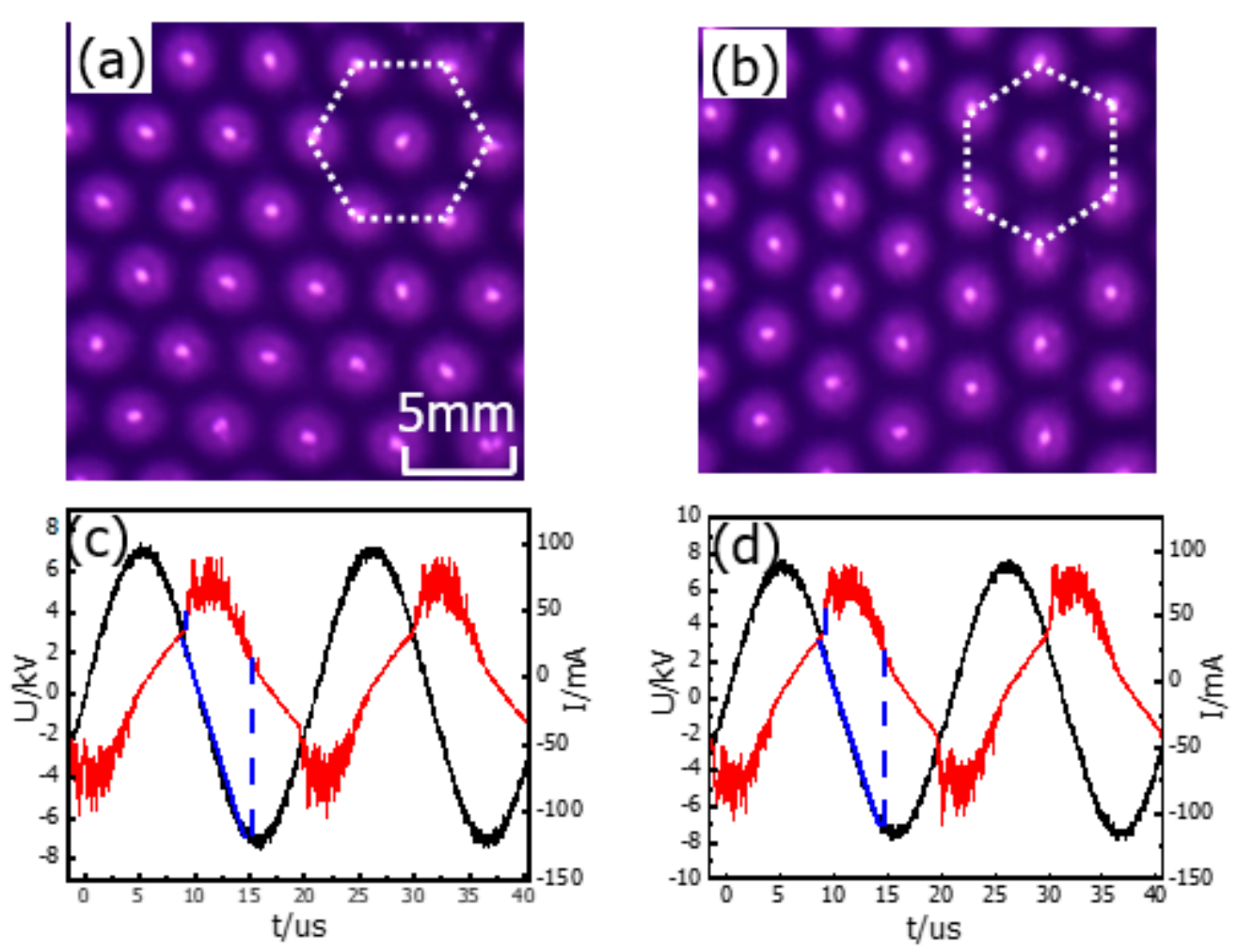

Figure 6. Reorientation of the triangular lattices when increasing of the applied voltage. $(\mathbf{a}, \mathbf{b})$ : snapshots of the plasma structures. (a) $U=7.4 \mathrm{kV}, p=52 \mathrm{~W}$ (b) $U=7.6 \mathrm{kV}, p=56 \mathrm{~W}$. The other discharge parameters are: $f=53 \mathrm{kHz}, p=440$ torr, $d=2.0 \mathrm{~mm}, t=40 \mathrm{~ms}$. The working gas is $65 \%$ Ar and $35 \%$ air. The white dotted hexagons indicate the angular reorientation of the plasma photonic crystals. (c,d): the corresponding voltage-current waveforms associate with the triangular lattices of different orientations. The blue lines on the voltage waveforms indicate the ranges of the voltages that the discharges occur.

\section{Conclusions}

In summary, we demonstrate a rich variety of structural tunable plasma photonic crystals by self-organization of the filaments in DBD. By changing the applied voltage, the plasma structures can be tuned flexibly in three ways, including changing the spatial symmetry, the lattice constant and the crystal orientation. The plasma structures changes from square lattice to triangular lattice, the lattice constant is reduced, and the crystal orientation varies $\frac{\pi}{6}$ when the applied voltage is increased. The band diagrams of the plasma photonic crystals under a TM wave are studied by using the finite element method, which shows that the positions and sizes of the band gaps change significantly for different 
plasma structures. A complete band gap is produced for the square plasma lattice, while a number of unidirectional band gaps are generated when the square lattice is switched to the triangular lattice. The photonic bands move towards higher frequencies by decreasing the lattice constant and the widths of the band gaps are increased. A flexible way to fabricate reconfigurable plasma photonic crystals is suggested, which may find wide application in the manipulation of microwaves or terahertz waves.

Author Contributions: The author K.G. performed the experiment measurements and wrote the paper. Y.L. and C.L. performed the simulations. W.F., F.L. and Y.H. discussed the results and revised the paper. All authors have read and agreed to the published version of the manuscript.

Funding: This work was supported by the National Natural Science Foundation of China (Grant Nos. 11875014, 11721091 and 11655002), the Natural Science Foundation of Hebei Province (Grant Nos. A2016201066 and A2017201099).

Conflicts of Interest: The authors declare no conflict of interest.

\section{References}

1. Yablonovitch, E. Inhibited Spontaneous Emission in Solid-State Physics and Electronics. Phys. Rev. Lett. 1987, 58, 2059. [CrossRef] [PubMed]

2. John, S. Strong Localization of Photons in Certain Disordered Dielectric Superlattices. Phys. Rev. Lett. 1987, 58, 2486. [CrossRef] [PubMed]

3. Alvarado-Beltrán, C.; Almaral-Sánchez, J.; Ramirez-Bon, R. Low temperature processing of multilayer dielectrics mirrors by sol-gel method. Mater. Lett. 2015, 161, 523. [CrossRef]

4. Yang, H.W.; Pan, L.; Han, Y.P.; Ma, L.H.; Li, Y.; Xu, H.B.; Zhao, J.P. A visual water vapor photonic crystal sensor with PVA/SiO $\mathrm{Si}_{2}$ opal structure. Appl. Surf. Sci. 2017, 423, 421. [CrossRef]

5. Lai, W.C.; Chakravarty, S.; Wang, X.; Lin, C.; Chen, R.T. On-chip methane sensing by near-IR absorption signatures in a photonic crystal slot waveguide. Opt. Lett. 2011, 36, 984. [CrossRef]

6. Pana, I.; Vitelaru, C.; Kiss, A.; Zoita, N.C.; Dinu, M.; Braic, M. Design, fabrication and characterization of $\mathrm{TiO}_{2}-\mathrm{SiO}_{2}$ multilayer with tailored color glazing for thermal solar collectors. Mater. Des. 2017, 130, 275. [CrossRef]

7. Paul, B.K.; Khalek, M.A.; Chalcma, S.; Ahmed, K. Chalcogenide embedded quasi photonic crystal fiber for nonlinear optical applications. Ceram. Int. 2018, 44, 18955. [CrossRef]

8. Suslik, L.; Pudis, D.; Goraus, M.; Nolte, R.; Kovac, J.; Durisova, J.; Gaso, P.; Hronec, P.; Schaaf, P. Photonic crystal and photonic quasicrystal patterned in PDMS surfaces and their effect on LED radiation properties. Appl. Surf. Sci. 2017, 395, 220. [CrossRef]

9. Monfareda, Y.E.; Ling, C.; Khosravi, R.; Kacerovska, B.; Yang, S. Selectively toluene-filled photonic crystal fiber Sagnac interferometer with high sensitivity for temperature sensing applications. Results Phys. 2019, 13, 102297. [CrossRef]

10. Monfared, Y.E.; Hajati, M.; Liang, C.; Yang, S.; Qasymeh, M. Quasi-D-Shaped Fiber Optic Plasmonic Biosensor for High-Index Analyte Detection. IEEE Sens. J. 2019, 1, 1. [CrossRef]

11. Fan, C.; Wang, J.; Zhu, S.; He, J.; Ding, P.; Liang, E. Optical properties in one-dimensional graded soft photonic crystals with ferrofluids. J. Opt. 2013, 15, 055103. [CrossRef]

12. Takeda, H.; Yoshino, K. Tunable photonic band schemes in two-dimensional photonic crystals composed of copper oxide high-temperature superconductors. Phys. Rev. B 2003, 67, 245109. [CrossRef]

13. Kitagawa, J.; Kodama, M.; Koya, S.; Nishifuji, Y.; Armand, D.; Kadoya, Y. THz wave propagation in two-dimensional metallic photonic crystal with mechanically tunable photonic-bands. Opt. Express 2012, 20, 17271. [CrossRef] [PubMed]

14. Chong, H.M.; De La Rue, R.M. Tuning of Photonic Crystal Waveguide Microcavity by Thermooptic Effect. IEEE Photonics Technol. Lett. 2004, 16, 1528. [CrossRef]

15. Yang, D.; Tian, H.; Ji, Y. Nanoscale photonic crystal sensor arrays on monolithic substrates using side-coupled resonant cavity arrays. Opt. Express 2011, 19, 20023. [CrossRef]

16. Park, W.; Lee, J.B. Mechanically tunable photonic crystal structure. Appl. Phys. Lett. 2004, 85, 4845. [CrossRef]

17. Guo, B. Photonic band gap structures of obliquely incident electromagnetic wave propagation in a one-dimension absorptive plasma photonic crystal. Phys. Plasmas 2009, 16, 043508. [CrossRef]

18. Shiveshwari, L.; Mahto, P. Photonic band gap effect in one-dimensional plasma dielectric photonic crystals. Solid State Commun. 2006, 138, 160. [CrossRef] 
19. Zhang, L.; Ouyang, J.T. Experiment and simulation on one-dimensional plasma photonic crystals. Phys. Plasmas 2014, 21, 103514. [CrossRef]

20. Zhang, H.F.; Liu, S.B.; Jiang, Y.C. Tunable all-angle negative refraction and photonic band gaps in two-dimensional plasma photonic crystals with square-like Archimedean lattices. Phys. Plasmas 2014, 21, 092104. [CrossRef]

21. He, X.; Chen, J.P.; Ni, X.W.; Chen, Y.D.; Zeng, X.J.; Gu, T.T. Numerical investigation on interference and absorption of Electromagnetic Waves in the Plasma-Covered Cavity Using FDTD Method. IEEE Trans. Plasma Sci. 2012, 40, 1010. [CrossRef]

22. Wang, B.; Cappelli, M.A. A plasma photonic crystal bandgap device. Appl. Phys. Lett. 2016, 108, 161101. [CrossRef]

23. Suthar, B.; Bhargava, A. Optical Properties of Plasma Photonic Crystals. Silicon 2015, 7, 433. [CrossRef]

24. Zhang, H.F.; Liu, S.B. The Tunable Omnidirectional Reflector Based on Two-Dimensional Photonic Crystals With Superconductor Constituents. IEEE J. Sel. Top. Quantum Electron. 2015, 21, 1-8. [CrossRef]

25. Zhang, H.F.; Liu, S.B.; Kong, X.K.; Zou, L.; Li, C.Z.; Qing, W.S. Enhancement of omnidirectional photonic band gaps in one-dimensional dielectric plasma photonic crystals with a matching layer. Phys. Plasmas 2012, 19, 022103. [CrossRef]

26. Tan, H.Y.; Jin, C.G.; Zhuge, L.J.; Wu, X.M. The SLR-Dependent Negative PBG in 1-D Plasma Photonic Crystal. IEEE Trans. Plasma Sci. 2019, 47, 3986. [CrossRef]

27. Sakaguchi, T.; Sakai, O.; Tachibana, K. Photonic bands in two-dimensional microplasma arrays. I. Theoretical derivation of band structures of electromagnetic waves. J. Appl. Phys. 2007, 101, 073304.

28. Liu, Y.Y.; He, F.; Zhao, X.F.; Ouyang, J.T. Evolution of Striation in Pulsed Glow Discharges. Plasma Sci. Technol. 2016, 18, 30. [CrossRef]

29. Khalkhali, T.F.; Bananej, A. Effect of shape of scatterers and plasma frequency on the complete photonic band gap properties of two-dimensional dielectric-plasma photonic crystals. Phys. Rev. A 2016, 380, 4092.

30. Chaudhari, M.K.; Chaudhari, S. Tuning photonic bands in plasma metallic photonic crystals. Phys. Plasmas 2016, 23, 112118. [CrossRef]

31. Chern, R.L.; Chang, C.C.; Chang, C.C. Analysis of surface plasmon modes and band structures for plasmonic crystals in one and two dimensions. Phys. Rev. E 2006, 73, 036605. [CrossRef] [PubMed]

32. Wang, B.; Cappelli, M.A. A tunable microwave plasma photonic crystal filter. Appl. Phys. Lett. 2015, 107, 171107. [CrossRef]

33. Sheng, Z.M.; Zhang, J.; Umstadter, D. Plasma density gratings induced by intersecting laser pulses in underdense plasmas. Appl. Phys. B 2003, 77, 673. [CrossRef]

34. Wu, H.C.; Sheng, Z.M.; Zhang, J. Chirped pulse compression in nonuniform plasma Bragg gratings. Appl. Phys. Lett. 2005, 87, 201502. [CrossRef]

35. Dong, L.; Yin, Z.Q.; Wang, L.; Fu, G.S.; He, Y.F.; Chai, Z.F.; Li, X.C. Square pattern formation in a gas discharge system. Thin Solid Films 2003, 435, 120. [CrossRef]

36. Fan, W.L.; Sheng, Z.M.; Dang, W.; Liang, Y.Q.; Gao, K.Y.; Dong, L.F. Spatiotemporally Controllable Plasma Lattice Structures in Dielectric Barrier Discharge. Phys. Rev. Appl. 2019, 11, 064057. [CrossRef]

37. Dong, L.; Fan, W.; He, Y.; Liu, F.; Li, S.; Gao, R.; Wang, L. Square superlattice pattern in dielectric barrier discharge. Phys. Rev. E 2006, 73, 066206. [CrossRef]

38. Matlis, E.H.; Corke, T.C.; Neiswander, B.; Hoffman, A.J. Electromagnetic wave transmittance control using self-organized plasma lattice metamaterial. J. Appl. Phys. 2018, 124, 093104. [CrossRef]

39. Dong, L.; Qi, Y.; Liu, W.; Fan, W. Measurement of the electron density in a subatmospheric dielectric barrier discharge by spectral line shape. J. Appl. Phys. 2009, 106, 013301. [CrossRef]

40. Dong, L.; Ran, J.; Mao, Z. Direct measurement of electron density in microdischarge at atmospheric pressure by Stark broadening. Appl. Phys. Lett. 2005, 86, 161501. [CrossRef]

41. Strümpel, C.; Purwins, H.G.; Astrov, Y.A. Spatiotemporal filamentary patterns in a dc-driven planar gas discharge system. Phys. Rev. E 2001, 63, 026409. [CrossRef] [PubMed]

42. Zamani, M.; Amanollahi, M.; Hocini, A. Photonic band gap spectra in Octonacci all superconducting aperiodic photonic crystals. Phys. B Condens. Matter 2019, 556, 151. [CrossRef]

(C) 2020 by the authors. Licensee MDPI, Basel, Switzerland. This article is an open access article distributed under the terms and conditions of the Creative Commons Attribution (CC BY) license (http://creativecommons.org/licenses/by/4.0/). 